\title{
Metacognição e seus 50 anos: cenários e perspectivas para o Ensino de Ciências
}

\author{
Cleci Teresinha Werner da Rosa*, Nancy Nazareth Gatzke Corrêa**, \\ Marinez Meneghello Passos ${ }^{\star * *}$, Sergio de Mello Arruda ${ }^{\star \star \star *}$
}

\section{Resumo}

Os estudos envolvendo metacognição completam 50 anos e revelam uma diversidade de áreas que tem utilizado esse conceito que foi cunhado por Flavell na década de 1970. Além disso, e mais próximo do contexto educacional, a metacognição tem sido apontada como uma proposta que contribui para a aprendizagem, o que tem oportunizado um olhar mais atento aos componentes metacognitivos (e seus respectivos elementos). Considerando a possibilidade de fazer um tributo a John Hurley Flavell que publicou seu primeiro artigo no ano de 1971 apresentando o termo metamemory que deu origem à denominação metacognição, elaboramos este artigo. Nele divulgamos um conjunto de estudos que abordam a utilização da metacognição em salas de aulas em Ciências, apresentando as características e os impactos dessas práticas, bem como trazendo novas possibilidades de estudos e intervenções. Para isso: introduzimos o termo historicamente e alguns detalhes a respeito das primeiras investigações realizadas; descrevemos diversas reflexões sugeridas por quem buscou avançar na compreensão e na aplicação da metacognição em inúmeras áreas; elencamos resultados de pesquisas ocorridas em salas de aulas; destacamos as possibilidades de escolhas e caminhos praticáveis para um Ensino de Ciências pautado em experiências metacognitivas.

Palavras-chave: John Hurley Flavell; Metacognição; Educação em ciências.

* Doutora em Educação Científica e Tecnológica pela Universidade Federal de Santa Catarina (UFSC). Docente do Programa de Pós-Graduação em Educação e do Programa de Pós-Graduação em Ensino de Ciências e Matemática da Universidade de Passo Fundo (UPF) - Passo Fundo, RS. Brasil. E-mail: cwerner@upf.br.

* Doutoranda do Programa de Pós-Graduação em Ensino de Ciências e Educação Matemática (PECEM) da Universidade Estadual de Londrina (UEL) - Londrina, PR. Docente da SEED, governo do Estado do Paraná. Brasil. E-mail: nancyngatzke@gmail.com.

.*. Doutora em Educação para a Ciência pela Universidade Estadual Paulista “Júlio de Mesquita Filho" (UNESP). Docente Sênior da Universidade Estadual de Londrina (UEL) - Londrina, PR - e docente Sênior colaboradora da Universidade Estadual do Norte do Paraná (UENP) - Câmpus Cornélio Procópio, PR. Brasil. E-mail: marinezpassos@uel.br. Com o apoio do CNPq.

**** Doutor em Educação pela Universidade de São Paulo (USP). Professor Sênior da Universidade Estadual de Londrina (UEL) - Londrina, PR. Brasil. E-mail: sergioarruda@uel.br. Com o apoio do CNPq.

Recebido em: 04/10/2020 - Aceito em: 03/03/2021

https://doi.org/10.5335/rbecm.v4i1.12194

http://creativecommons.org/licenses/by-nc-nd/4.0 


\section{Introdução}

A metacognição tem sido uma área de interesse para pesquisadores do campo da Educação desde a década de 1980, todavia, seus primeiros estudos datam do início dos anos de 1970, mais precisamente no ano de 1971, quando o psicólogo americano John Hurley Flavell publicou o artigo intitulado "First discussant's comments: what is memory development the development of?" - que em 2021 completa 50 anos. Todavia o termo utilizado naquela ocasião foi "metamemory", ou seja, "metamemória", mas que na continuidade deu origem à denominação "metacognição".

Desde então pesquisadores do campo da Psicologia e mais tarde do campo educacional vêm se servindo das discussões oportunizadas por Flavell e outros pesquisadores engajados em ampliar e aplicar esse entendimento em diversas situações de aprendizagem. A publicação de Flavell, no ano de 1976, intitulada "Metacognitive aspects of problem solving" e o Modelo de Monitoração Cognitivo, criado por ele em 1979, são considerados pelos investigadores da área 'marcos' dos estudos envolvendo metacognição, especialmente em sua aproximação com os processos de aprendizagem.

Tais publicações trouxeram o entendimento de que a metacognição "se refere ao conhecimento que se tem dos próprios processos e produtos cognitivos ou de qualquer outro assunto relacionado a eles, por exemplo, as propriedades relevantes para a aprendizagem de informações ou dados" (FLAVELL, 1976, p. 232, tradução nossa). Conceito que sofreu e vem sofrendo alterações especialmente a partir das aproximações que tem sido realizada em campos específicos do conhecimento, como é o caso das diferentes áreas da Educação. Entretanto, embora tenhamos uma variação de seu conceito é possível conceber que há um núcleo coeso entorno do qual os pesquisadores estabelecem suas definições. Esse núcleo está vinculado à definição dada por Flavell e, por isso, ele é considerado o precursor, em se tratando dos estudos envolvendo metacognição, independentemente da área em estudo.

Sociologia, Educação, Psicologia, Linguística, entre outras áreas, vem se servindo desse entendimento e estabelecendo suas relações com o conceito de modo a oportunizar um debate sobre como podemos levar as pessoas a utilizar esse recurso de pensamento como uma estratégia para lograr êxito em suas mais distintas atividades. No campo educacional e na especificidade dos processos de aprendizagem, a metacognição tem sido apontada como um dos diferenciais na estrutura de 
pensamento daqueles que apresentam facilidade de aprendizagem (os experts) em comparação àqueles considerados com dificuldade (novatos). Hattie (2009), ao elencar um conjunto de aspectos que se revelam mais significativos para a aprendizagem mostra que a utilização do pensamento metacognitivo está entre os principais.

Weinert (1987) menciona que a metacognição, em seu sentido mais geral, pode ser entendida como um pensamento do pensamento ou uma cognição de segunda ordem. Hacker (1998), mostra que esse pensamento pode ser do que alguém sabe (conhecimento cognitivo), do que alguém está atualmente fazendo (habilidade metacognitiva), ou do estado afetivo ou cognitivo atual de alguém (experiência metacognitiva). No entender do autor para diferenciar o pensamento metacognitivo de outros tipos de pensamento, é necessário considerar a fonte dos pensamentos metacognitivos, ou seja, que os pensamentos metacognitivos não surgem de uma realidade externa imediata da pessoa, mas sim, das representações mentais internas próprias dessa pessoa sobre aquela realidade, a qual pode incluir o que alguém sabe sobre essa representação interna, como ela funciona, e como alguém se sente sobre ela. Portanto, a metacognição pode ser entendida como "conhecimento e cognição sobre o fenômeno cognitivo" (FLAVELL, 1979, p. 906).

Nesse viés, autores como a psicóloga americana Ann Lesley Brown - outra pesquisadora com significativa contribuição nos estudos envolvendo metacognição - definem-na com a consciência do próprio conhecimento ou a compreensão desse conhecimento, concebendo que o sujeito, ao compreender esse conhecimento, poderá utilizá-lo adequadamente na realização das atividades (BROWN, 1978). Para a autora deve-se associar metacognição, em um primeiro momento, ao conhecimento sobre os recursos e as estratégias mais adequadas à efetivação de determinada tarefa - o conhecimento do conhecimento; em outro, ao controle executivo, que abrange mecanismos autorregulatórios quando da realização da tarefa, como a planificação, a monitoração e a avaliação das realizações cognitivas. Ambos (os momentos) estão inter-relacionados, cada um alimentando o outro, embora possam ser facilmente identificáveis.

Tais entendimentos tem subsidiado nossa dedicação ao tema nos últimos anos e tem revelado que existe grande quantidade de divulgações literárias sobre metacognição, tanto teóricas quanto empíricas, entretanto, poucas são os estudos que apresentam práticas instrucionais específicas para provocar nos alunos o aprendizado metacognitivo. Ou seja, temos uma quantidade significativa de artigos 
produzidos ao longo desses 50 anos, mesmo se considerarmos um recorte relativo à área de Ensino de Ciências, mas poucos são os estudos que retratam práticas instrucionais que possibilitem ao professor agregá-las em suas ações pedagógicas. O que pode ser identificado - e que nos ocuparemos disso no presente artigo -, são estudos específicos ou clínicos que fornecem indicativos de que ao serem utilizadas essas práticas no contexto escolar elas qualificam a aprendizagem em determinados componentes curriculares, por meio da promoção de uma "reflexão metacognitiva" ou, alternativamente, mostram-se limitantes a isso.

O crescente número de artigo na área de Ensino de Ciências foi apontado por Zohar e Barzilai (2013) ao mapear 178 estudos publicados no período de 2000 a 2012 na base de dados Educational Resources Information Center (ERIC). As autoras concluíram que embora tenha sido percebido um aumento no interesse da área pela temática, muito ainda há para se pesquisar, especialmente, em relação às práticas de intervenção didática. Situação que igualmente foi revelada na revisão de literatura não sistemática realizada por Avargil, Lavi e Dori (2018), mostrando que as pesquisas estão centradas no uso de estratégias metacognitivas, treinamento metacognitivo e avaliação da aprendizagem. Para esses autores, assim como para outros da área, as pesquisas em metacognição associados ao Ensino de Ciências podem contribuir, significativamente, para o desenvolvimento do pensamento crítico, autonomia e resolução de problemas complexos.

O modo como os estudos nessa área vem se servindo da metacognição e os resultados obtidos são objetos de discussão do presente texto que apresenta como objetivo relatar sobre um conjunto de investigações que tem se ocupado de levar a metacognição para o contexto das aulas de Ciências (Física, Química e Biologia), apresentando as características e impactos dessas práticas, bem como trazer novas possibilidades de intervenções. Foge ao escopo do artigo realizar uma revisão sistemática do conceito ou mesmo das pesquisas envolvendo ações pedagógicas e metacognição, mas, ao invés disso, o texto busca possibilidades e pretende servir de inspiração para novos trabalhos, fomentando sua inserção como estratégia de ensino e de aprendizagem.

Justificamos nossa intenção enquanto possibilidade de ampliação das práticas voltadas à promoção do pensamento metacognitivo em sala de aula, tanto na pesquisa, como na ação docente, considerando especialmente sua escassez (nas divulgações), o que representa uma lacuna para a área. Além disso, mencionamos que o 
texto tem caráter comemorativo em alusão aos 50 anos da publicação do primeiro artigo no ano de 1971, por John Hurley Flavell, como relatado anteriormente.

Para tanto, na composição deste artigo, apresentamos reflexões sobre o entendimento de metacognição, sem, contudo, atermo-nos, em demasia, nessa discussão; na sequência relatamos a respeito de estudos vinculados a intervenções didáticas e seus resultados, tendo como foco trazer elementos que permitam discutir a presença da metacognição na área de Ensino de Ciências.

\section{Reflexōes sobre metacognição: alguns destaques}

Martinez (2006) menciona que a descoberta e a elaboração teórica da metacognição constituem um grande avanço nas últimas décadas de pesquisa cognitiva, particularmente em se tratando da aprendizagem. Todavia, o autor chama a atenção para o fato de que o conceito que vem sendo utilizado não é definitivo e necessita estar constantemente sendo revisado a partir do avanço dos estudos. Nessa perspectiva admitimos a falta de consenso sobre um conceito único e capaz de abarcar todas as áreas e especificidades, inerentes a elas, que envolvem a metacognição, ressaltando sua complexidade. Por outro lado, apontamos que os estudos desenvolvidos já são suficientemente capazes de estabelecer que: "Metacognição é o conhecimento que o sujeito tem sobre seu conhecimento e a capacidade de regulação dada aos processos executivos, somada ao controle e à orquestração desses mecanismos" (ROSA, 2011, p. 57). Nesse entendimento estariam presentes os componentes metacognitivos que têm sido considerados por um grupo de pesquisadores como componentes do conceito, quais sejam: experiências metacognitivas, conhecimentos metacognitivos e habilidades metacognitivas.

De acordo com Rosa (2011) e retomando Flavell (1979), temos que as experiências metacognitivas estariam associadas às impressões ou percepções conscientes que podem ocorrer antes, durante ou após a realização de uma ação ou sempre que o sujeito vivenciar alguma dificuldade ou falta de compreensão de algo de grande importância para ele. Pode-se, então, chegar aos meios de sua superação. As experiências metacognitivas são conscientes, cognitivas e afetivas; podem ser breves ou longas, simples ou complexas, em termos de conteúdos; também podem servir para uma variedade de funções úteis nas iniciativas cognitivas. Os conhecimentos metacognitivos, por sua vez, referem-se aos conhecimentos que o sujeito tem so- 
bre si próprio no que se refere às variáveis pessoa, tarefa e estratégia e, também, à maneira como essas variáveis interferem no resultado da cognição. Por fim, as habilidades metacognitivas estão relacionadas à capacidade do sujeito de regular e controlar sua ação, por meio da operação de planejamento, monitoramento da ação e avaliação do executado.

Tais componentes e seus respectivos elementos têm sido apontados como associados ou integrantes do processo de reflexão metacognitiva e que no entender de Rosa (2011), necessitam estar presentes como estratégias para que os alunos logrem êxito em suas atividades de aprendizagem. Embora possamos dizer que esses componentes podem fornecer um marco para estabelecer uma ampliação do conceito inicial proposto por Flavell, precisamos considerar que pesquisadores como Nelson e Narens (1994), por exemplo, consideram que as componentes podem ser expressas na monitoração e no controle. Sem entrar na enseada das diferentes possibilidades conceituais, pois isso seria um artigo específico, mencionamos que há uma significativa gama de possibilidades de estabelecer tais componentes, todavia, as apresentadas têm sido alimentadas por estudos que se dedicam a trazer a metacognição para situações de sala de aula, mesmo que ainda timidamente e com pouco detalhamento sobre a presença dessas componentes, como veremos ao longo do artigo.

Apesar das diferentes possibilidades de entender a metacognição, seus componentes e elementos, é factível constatar os benefícios de sua utilização no campo educacional desde os primeiros estudos desenvolvidos por Flavell na década de 1970, cujos resultados apontados são promissores, especialmente sobre o desempenho dos estudantes no campo da leitura e da resolução de problemas. Investigações envolvendo práticas pedagógicas que enfatizam a metacognição como elemento estruturador do pensamento evidenciam que a melhoria na aprendizagem está relacionada ao fato de que esse pensamento leva ao desenvolvimento de habilidades como as associadas à leitura, à resolução de problemas e ao pensamento de ordem superior.

\section{Estudos envolvendo a metacognição em sala de aula}

Dentre as pesquisas que associam a metacognição às situações didáticas no campo da Educação Científica e que tem revelado benefícios à aprendizagem dos estudantes, os resultados mais promissores têm sido daqueles que apresentam uma 
alteração na instrução de ensino em função da metacognição, envolvendo momentos explícitos de ativação desse pensamento no contexto da disciplina (AVARGIL; LAVI; DORI, 2018). Estudos como os realizados por Grotzer e Mittlefehldt (2012) são exemplos de intervenções em que o contexto da disciplina foi alterado, de modo a inserir ações que oportunizassem a evocação do pensamento metacognitivo de forma explícita. Esse estudo procurou modificar a disciplina de Ciências do oitavo ano, não se limitando a vincular a metacognição a uma ação ou situação didática específica (leitura de textos, resolução de problemas, atividades experimentais, simulação, etc.). A intervenção, orientada por material instrucional desenvolvido pelo professor, teve como objetivo encorajar os alunos a monitorar e avaliar suas ações, possibilitando espaços de discussão em sala de aula. Os resultados demonstram uma correlação direta entre as manifestações de natureza metacognitiva feita pelos estudantes durante as atividades e os resultados do pós-teste que foi de conhecimento do conteúdo.

Todavia, esse estudo é atípico, pois grande parte das investigações se limita a inserir momentos de ativação do pensamento metacognitivo associado a uma atividade didática e a partir dela analisar a viabilidade e os benefícios logrados para a aprendizagem. Tais estudos têm revelado resultados que apontam a possibilidade e a necessidade de associar às disciplinas curriculares situações que promovam a ativação do pensamento metacognitivo, embora para alguns estudantes essa ativação possa ser realizada de forma espontânea, sem necessidade de ser favorecida por alguma instrução ou realização de tarefa, como veremos mais adiante.

Os estudos área de Ensino de Ciências, ainda que realizados em número reduzido, voltados a investigar uma ação didática específica e, por vezes, direcionados a avaliar a metacognição de forma mais geral (reflexão metacognitiva), apontam que as situações orientadas a ativar de forma explícita o pensamento metacognitivo nos estudantes revelam melhoria em aspectos como: compreensão dos conceitos e fenômenos (EILAM; REITER, 2014; GUNSTONE, 1994; ROSA, 2011); motivação para aprender (TAASOOBSHIRAZI; FARLEY, 2013; THOMAS, 2013); autoconfiança do aprendiz (HUFF; NIETFELD, 2009; VERPOORTEN et al., 2014); revisão em crenças epistemológicas (HUANG; GE; ESERYEL, 2017; KALMAN et al., 2015; PETERS-BURTON, 2012; YURUK, 2007); melhoria na leitura de textos científicos (KOCH, 2001; WANG; CHEN, 2014); aumento da capacidade de resolução de problemas (ANDERSON; NASHON, 2007; NETO; VALENTE, 1997; NIELSON; 
NASHON; ANDERSON, 2009; RYAN et al., 2016; TAASOOBSHIRAZI; FARLEY, 2013;); análise da argumentação de estudantes em atividade experimental de Biologia (MEDEIROS; SILVA; LOCATELLI, 2018); estabelecimento de categorias metacognitivas a partir de entrevistas com estudantes de Ensino Médio (PASSOS; CORREAA; ARRUDA, 2017; CORRÊA; PASSOS; ARRUDA, 2018a, 2018b); oportunizar o desenvolvimento do pensamento crítico (TAMAYO-ALZATE, 2006). Na continuidade, passamos a descrever alguns desses estudos, contribuindo com o que idealizamos e objetivamos com a elaboração deste artigo.

No estudo de Rosa (2011) foi analisada a viabilidade de uma proposta didático-metodológica para as atividades experimentais em Física, que explicitasse momentos de ativação do pensamento metacognitivo. Para tanto, a autora propôs uma estrutura de aulas experimentais guiadas por questionamentos metacognitivos e orientadas a ativar elementos metacognitivos (pessoa, tarefa, estratégia, planificação, monitoração e avaliação). O modelo foi testado no sistema educacional brasileiro em uma turma de primeiro ano do Ensino Médio, com 12 estudantes, apresentando como resultado sua viabilidade didática. Em termos metacognitivos o estudo evidenciou que o modelo proposto é favorecedor da ativação dos seis elementos metacognitivos elencados para o estudo, contudo, alguns são mais difíceis de serem ativados pelos estudantes ou avaliados na proposta (estratégia e monitoramento).

O estudo desenvolvido por Taasoobshirazi e Farley (2013) propôs um modelo de resolução de problemas de Física que envolvia entre outros aspectos os de natureza metacognitivas. Este modelo buscou relacionar variáveis utilizadas pelos estudantes que apresentassem facilidade na aprendizagem em Física, incluindo: motivação, planejamento metacognitivo, uso de estratégia, habilidades de categorização, elaboração de diagramas de corpo livre. Participaram da pesquisa de forma voluntária 125 estudantes dos estados da Geórgia e de Nevada, dos Estados Unidos da América, e que estavam matriculados em uma disciplina de Física de nível introdutório em cursos de graduação em Ciências, Física e Engenharia. Foi solicitado aos estudantes, que de forma individual, respondessem um conjunto de atividades que incluía, na ordem: um questionário de motivação, questões avaliando o planejamento metacognitivo, cinco problemas de Física projetados para avaliar o uso da estratégia, construção de diagramas de corpo livre sobre o problema, pontuações da solução de problemas, quatro tarefas de categorização para avaliar se os alunos estavam se concentrando nas características conceituais dos problemas de Física. Os resultados indicaram 
que a motivação e a capacidade de categorização das etapas do desenvolvimento da resolução do problema, influenciaram a ativação do pensamento metacognitivo traduzido no estudo por meio do uso de estratégias metacognitivas. Essas, por sua vez, tiveram impacto direto nas pontuações atribuídas à resolução dos problemas em Física. Além de mostrar a importância das estratégias metacognitivas para a resolução de problemas em Física, o estudo ressaltou a necessidade de que os professores atribuam um tempo significativo para que os alunos compreendam os problemas de forma conceitual, avaliem as estratégias selecionadas, corrigindo-as sempre que necessário e sintam-se motivados para sua resolução e, somente depois, partam para ela.

Verpoorten et al. (2014) analisaram as contribuições de um game para desenvolver conhecimento em Óptica Geométrica e para estimular o desenvolvimento de habilidades metacognitivas a partir da capacidade de autoavaliar o grau de confiança em si mesmo. O jogo comercial (Elektra) foi utilizado com 28 estudantes do Ensino Médio na França e envolvia momentos explícitos de ativação do pensamento metacognitivo, particularmente, em relação às habilidades (planejamento, monitoração e avaliação) e confiança em si mesmo. Os resultados do estudo medidos por meio de questionários cognitivos e metacognitivos apontaram poucos ganhos cognitivos (apropriação dos conteúdos de Óptica), mas significativos avanços no uso do pensamento metacognitivo. A atividade realizada com o jogo mostrou que a inserção de momentos de reflexão metacognitiva não prejudica ou altera o fluxo do jogo, desde que certas condições sejam respeitadas, podendo ser utilizada sem prejuízo aos objetivos do jogo anunciados por seu fabricante.

Como possibilidade de discutir as crenças epistemológicas dos estudantes mencionamos o estudo de Kalman et al. (2015), que se baseou na hipótese de que essas crenças poderiam tornar-se mais experientes com uma combinação de atividades instrucionais apropriadas: leitura prévias com reflexão metacognitiva; aprendizagem ativa em classe que produz dissonância cognitiva. A hipótese foi testada por meio de um estudo de cinco anos em quatro cursos de Física de duas instituições em Montreal e Calgary, no Canadá, envolvendo cerca de 1000 alunos. Para o estudo foram utilizadas entrevistas com os estudantes, avaliações da escrita e Questionário de Crenças Epistemológicas Focalizadas (Discipline-Focused Epistemological Beliefs Questionnaire - DFEBQ). Por meio do questionário e a partir do desenho metodológico das atividades na disciplina de Física, os autores relataram que as 
crenças de aprendizes novatos tornaram-se menos ingênuas em dois dos quatro fatores analisados. Por fim, os autores inferiram que a combinação de atividade que leva os alunos a examinar material textual de forma metacognitiva (Escrita Reflexiva), com um ou mais tipos de intervenções de aprendizagem ativa na classe, pode promover mudanças positivas nas crenças epistemológicas dos alunos.

Sandi-Urena, Cooper e Stevens (2012), desenvolveram um estudo para analisar o efeito das aulas de laboratório em Química no modelo cooperativo e baseado em projetos, em termo de resolução de problemas e de desenvolvimento de habilidade metacognitiva. Para tanto, realizaram na disciplina de Química Orgânica um conjunto de aulas de laboratório com estudantes universitários em grupos de quatro participantes. Cada grupo recebia a situação problema e deveria resolvê-la por meio do trabalho colaborativo que caracteriza a abordagem por projetos. Ao final cada estudante entregava uma espécie de memória que foi considerado um dos instrumentos de avaliação das atividades. Para cada uma das aulas foram propostas atividades que exigiam planejamento, monitoração e avaliação, elementos identificados no estudo como associados às habilidades metacognitivas. Os resultados evidenciaram o efeito positivo do laboratório cooperativo baseado em projetos na resolução de problemas e na evocação do pensamento metacognitivo.

Saribaş, Mugaloğlu e Bayram (2013) analisaram a influência da orientação metacognitiva em futuros professores de Ciências durante as aulas de laboratório voltadas a discutir o conhecimento processual, declarativo e conceitual dos conteúdos de conhecimento científico, habilidades de processos científicos como: observação, experimentação, produção de dados; interpretação; visão da Natureza da Ciência. A investigação utilizou prompts metacognitivos que incluiam perguntas, discussões e reflexões durante as atividades experimentais e que tinham como objetivo ativar o pensamento metacognitivo nos estudantes. O estudo foi desenvolvido com 48 estudantes universitários do primeiro ano do curso de Química de uma universidade pública da Turquia, envolvendo dois grupos, um experimental e outro de controle. Para a produção dos dados foi utilizado pré e pós-testes e relatórios dos estudantes. Os resultados indicaram que ambos os grupos mantiveram as mesmas concepções equivocadas sobre a Natureza da Ciência, todavia, o grupo experimental que envolvia orientação metacognitiva para o desenvolvimento das atividades, manifestou maior compreensão conceitual, desenvolvimento de habilidades do método científico e emprego de estratégias metacognitivas de aprendizagem. 
No contexto brasileiro, a pesquisa de Medeiros, Silva e Locatelli (2018) visou por meio de um experimento que, consistia em colocar uma planta em dois tubos contendo água com fenolftaleína, onde um ficaria em uma câmara escura e outro em um ambiente com claridade, e por meio deste, aplicar conhecimentos de Biologia e desenvolver habilidades argumentativas. Foi solicitado aos alunos prever o que aconteceria com cada tubo e comparar com as mudanças na cor do $\mathrm{pH}$. Ao vivenciar uma atividade experimental sobre fotossíntese e respiração baseada na Previsão-Observação-Argumentação, foi possível analisar incidentes metacognitivos presentes no discurso dos estudantes: confirmação, monitoramento, mudança positiva e mudança negativa, e concluíram o estudo sinalizando que atividades envolvendo a argumentação favoreceram a capacidade de autorregulação do processo de aprendizagem.

Também em pesquisas nacionais aplicadas no contexto do Ensino Médio, os estudos de Passos, Côrrea e Arruda (2017) e Corrêa, Passos e Arruda (2018b), buscando analisar as percepções e reflexões a respeito do processo de aprendizagem em Física de estudantes do Ensino Médio, por meio de entrevista, elaboraram um instrumento de análise que permitisse traçar perfis metacognitivos de aprendizagem em Física, em que buscavam evidenciar as metas e as ações cognitivas por meio de categorias: especificidade do conhecimento; associativa; contextual; estratagema; constatação; processo cognitivo; memorização; experimentos escolares; sentimento de entendimento; interesse; emoções confortáveis; emoções desconfortáveis; comparação com o outro; necessidade do outro; relação com o mundo. Ao ser aplicado na análise das falas dos entrevistados, foi possível compreender que os estudantes conseguiram perceber sua aprendizagem em Física e foi estabelecido o perfil metacognitivo da aprendizagem em Física dos estudantes entrevistados.

Os mesmos autores, supracitados, em um movimento de pesquisa posterior (CORRÊA et al., 2020), realizaram um mapeamento da utilização da palavra e do conceito 'metacognição', considerando periódicos da área de Ensino de Ciências, no âmbito nacional, com estrato A1 e A2 designados pela Coordenação de Aperfeiçoamento de Pessoal de Nível Superior (CAPES). Entre as considerações conclusivas a que chegaram estão: as grandes áreas de utilização do conceito - Processo de Aprendizagem; Processo Metacognitivo; Formação de Professores; Instrumento de Avaliação; Objeto de Aprendizagem; Ambiente de Aprendizagem. Além disso, por meio da significação da palavra metacognição nas frases em que foram menciona- 
das nas referidas publicações, foi possível elaborar uma proposta de convergência representacional do termo "metacognição", assim organizada e especificada: Processo Metacognitivo; Estratégia Metacognitiva; Habilidade Metacognitiva; Caráter Metacognitivo; Pensamento Metacognitivo; Aprendizagem; Conhecimento Metacognitivo; Metacognição Docente; Autorregulação; Tomada de Consciência; Competência Metacognitiva; Modelo Metacognitivo; Nível Metacognitivo.

Como já mencionamos, nesse conjunto de estudos e resultados apresentados e que se encontram vinculados a intervenções realizadas na área de Ensino de Ciências, prioritariamente, assim como em outras, a ênfase está em incluir momentos explícitos de evocação metacognitiva em consonância com as atividades estruturadas para a disciplina, promovendo o que se denomina de "evocação explícita do pensamento metacognitivo". As considerações finais dessas investigações (neles apresentadas) apontam que a alteração na ação pedagógica oportuniza a reflexão que leva a uma ação controlada e consciente dos estudantes sobre seus conhecimentos e procedimentos. Ainda que teçamos críticas a essa forma simplista e ingênua que muitos estudos têm em relação à metacognição, consideramos que essa tem sido a forma encontrada pelos pesquisadores para evidenciar sua presença, embora alguns já tenham avançado e oportunizado um olhar mais atento aos componentes metacognitivos (e seus respectivos elementos), como relatado anteriormente.

\section{Metacognição: escolhas, caminhos, desejos...}

O mencionado nas seções passadas explicita que a reflexão metacognitiva ocorreu graças à oportunidade dada pelo professor para que isso se concretizasse. Monereo (2001), um dos defensores da importância de ativar o pensamento metacognitivo por meio de instruções explicitamente orientadas a isso, destaca a importância do professor como responsável por essa ativação ou modelo a ser seguido pelos estudantes. De acordo com o autor, além de mostrar que são metacognitivos, os professores precisam enaltecer os momentos em que essa forma de pensamento está presente. Isso ocorre, em seu entendimento, por meio de ações que contemplem situações orientadas a essa evocação e não pode ser entendido como uma instrução a parte e específica sobre metacognição.

Em consonância com o que aponta por Monereo (2001), temos o estudo de Kistner et al. (2010), realizado na Alemanha, envolvendo 20 professores de Matemática. 
De acordo com a investigação, aqueles que utilizaram estratégias metacognitivas explícitas, reorientando suas ações pedagógicas, apresentaram uma eficácia de ensino maior em comparação com aqueles que a usaram implicitamente, ou seja, que recorreram a estratégias sem momentos específicos de ativação dessa forma de pensamento.

Todavia, a literatura tem se ocupado de analisar também situações em que não há uma orientação explícita, mas que se utiliza de ações que em sua concepção teórica mostra uma potencialidade metacognitiva. Esse é o caso dos mapas conceituais, "V" epistemológico de Gowin, estratégia POE (Predizer-Explicitar-Explicar) e diários de aprendizagem. No caso dos diários de aprendizagem, ação escolhida para exemplificar esses estudos, temos o trabalho de Boszko e Rosa (2020), que avaliaram as contribuições dessa ação didática para a ativação do pensamento metacognitivo. Sem alterar o modo como esses recursos eram utilizados no contexto de aprendizagem, as autoras analisaram os diários produzidos por oito acadêmicos de um curso de licenciatura em Física de uma universidade brasileira, durante 13 encontros consecutivos. As autoras concluíram que eles oferecem uma potencialidade metacognitiva, mesmo não estando orientados a isso. Todavia, as pesquisadoras chamam a atenção para o fato de que, se houvesse uma orientação mais direta sobre como ativar essa forma de pensamento, os resultados poderiam ter sido mais promissores, inferindo, ao final do estudo, a necessidade de incluir uma orientação para potencializar o uso dos diários com ativador do pensamento metacognitivo. Tal inferência corrobora com a recomendação de Monereo (2001), no sentido de que, para ativar essa forma de pensamento, é preciso inserir momentos explícitos voltados a isso.

Seguindo as indicações relativas a uma orientação direta para ativar o pensamento metacognitivo, entra em cena as proposições pedagógicas, que podem ser planejadas pelos professores, o que nos remete a dois aspectos podem ser contemplados em se tratando dos estudos que envolvem a metacognição e o contexto escolar relacionado ao ensino de Ciências. Um deles consiste nas diferenças na estrutura de pensamento metacognitivo dos sujeitos que são considerados experts em determinadas disciplinas e dos que são considerados novatos ${ }^{1}$. Outro aspecto é a tendência das pesquisas atuais em envolver discussões sobre o papel das interações sociais, do contexto e do ambiente, em que os estudantes estão inseridos para a utilização do pensamento metacognitivo - a metacognição social. Nesse último caso, assume 
destaque o papel da argumentação e da aprendizagem colaborativa. Passamos a discutir esses aspectos, relatando pesquisas que têm se ocupado de analisá-los.

No caso dos estudantes com expertise, estudos como os desenvolvidos por Chi e colaboradores desde a década de 1980 (CHI; GLASER; REES, 1982; CHI et al., 1989) vêm mostrando que o diferencial desses indivíduos em relação ao que apresentam dificuldades para compreender determinados conteúdos está no uso espontâneo do pensamento metacognitivo. Por exemplo, o estudo empreendido por Bogdanovic et al. (2017) na República Sérvia revelou a existência de uma correlação direta entre a consciência metacognitiva e os resultados em Física. Os autores analisaram o desempenho de 746 estudantes na faixa etária de 15 anos por meio de questionários relacionados ao uso do pensamento metacognitivo e teste de conhecimentos específicos de Física. Como resultado, apontaram haver diferenças no desempenho em Física de alunos que apresentaram maior consciência metacognitiva, distinto do revelado pelos que não utilizaram essa forma de pensamento.

Larkin (1983) e Schraw, Crippen e Hartley (2006), por seu turno, demonstraram que as diferenças entre experts e novatos está, entre outros aspectos, no modo como os primeiros estruturam a solução do problema. Para os autores, os experts destinam um tempo significativo ao planejamento, enquanto que os novatos partem de imediato para a coleta de dados e escolha da fórmula. Os experts de acordo com esses estudos, partem para a solução somente depois de terem clareza da representação desse problema ou da situação envolvida. Taasoobshirazi e Farley (2013, p. 53, tradução nossa) resumem a diferença entre experts e novatos mencionando que:

[...] os experts usam mais estratégias orientadas para a meta ao resolver problemas, tem um conhecimento maior e mais organizado, são mais metacognitivos, têm maior motivação e tendem a receber mais apoio social (por exemplo, Alexander, 2003; Ericsson, 2006; Hatano \& Oura, 2003; Zimmerman, 2006).

Em termos da perspectiva social como elemento vinculado à ativação do pensamento metacognitivo em processos de intervenção pedagógica, destaca-se que, embora a metacognição possa ser vislumbrada sob o ponto de vista de uma avaliação voltada para dentro, especialmente às ideias e aos pensamentos do próprio estudante, precisamos considerar nesse processo a influência da componente social e cultural vinculada ao contexto de inserção desse sujeito. Como referencial, podemos pensar nos estudos de Vygotsky, os quais nos mostram que o pensamento de ordem superior começa como discurso social e que esses padrões de discurso são internalizados ao 
longo do tempo e da experiência. As experiências, por sua vez, estão relacionadas com o contexto e contribuem para moldarmos pensamentos e ações.

A importância da ativação do pensamento metacognitivo por mecanismos decorrentes da verbalização de ideias, argumentação e interação entre sujeitos, típicos das relações sociais existentes em um contexto de aprendizagem, já havia sido tratada por Flavell em 1979. Nas palavras do autor:

Os investigadores concluíram recentemente que a metacognição desempenha um
papel importante na comunicação oral de informação, persuasão oral, compreensão
oral, compreensão de leitura, escrita, aquisição de linguagem, atenção, memória,
resolução de problemas, cognição social e vários tipos de autocontrole e autoinstru-
ção; também há indícios claros de que as ideias sobre metacognição estão começando
a entrar em contato com ideias semelhantes nas áreas da teoria da aprendizagem
social, modificação do comportamento cognitivo, desenvolvimento da personalidade
e educação (FLAVELL, 1979, p. 906, tradução nossa).

No artigo, o autor parte das contribuições da metacognição para a verbalização e a argumentação de ideias, o que também ocorre no seu revés. Ou seja, a verbalização e a argumentação fomentam a organização e o revistar de ideias que podem oportunizar a ativação desse tipo de pensamento. Phillips, Watkins e Hammer (2018) mostram que, nesse processo de revistar um conhecimento que muitas vezes está posto como consolidado pela comunidade científica, ou, no caso da escola, um conhecimento que julgamos ter aprendido, podemos encontrar lacunas e incoerências desse conhecimento, de modo a proceder com uma reflexão de natureza metacognitiva.

Os estudos de Thomas e Mee (2005), Nielsen, Nashon e Anderson (2009) e Thomas (2013) são exemplos de trabalhos que consideram a vertente social como favorecedora da ativação do pensamento metacognitivo. Tais estudos apontam para a compreensão de que a natureza social do fenômeno educativo, envolvendo aspectos interacionistas, históricos, sociológicos, culturais, entre outros, leva a uma nova compreensão de metacognição, em que a habilidade de regular e controlar o pensamento representa um processo dependente da cultura, aprendido socialmente, não construído exclusivamente a partir de situações didáticas voltadas ao domínio dos conteúdos.

O estudo de Thomas e Mee (2005), por exemplo, evidencia que a metacognição é socialmente mediada, oferecendo aos alunos jovens uma oportunidade para falar sobre seus processos de aprendizagem e pensamento. Os autores relatam sobre o impacto de uma intervenção em sala de aula de dois meses e que teve por objetivo 
alterar o ambiente de aprendizagem incluindo ações didáticas de natureza metacognitiva, em duas salas de aula do Ensino Fundamental em Hong Kong, na China. Para coleta dos dados foi empregado uma abordagem envolvendo dados quantitativos com pré e pós-intervenção (General Studies Metacognitive Orientation Scale - GSMOS) e qualitativas (entrevistas com os alunos e observações em sala de aula). Embora os testes não tenham demonstrado diferenças estatísticas, as entrevistas e a observação forneceram dados que permitiram aos autores inferir que a intervenção pedagógica contribuiu para o desenvolvimento de conhecimento metacognitivo, bem como para a conscientização e o controle no uso das estratégias em salas de aula. Tais resultados foram atribuídos ao modo como as atividades foram organizadas, privilegiando trocas e diálogos entre os participantes.

Essa perspectiva é corroborada por Anderson e Nashon (2007), ao apontarem que, na aprendizagem em Física, as situações problema do tipo aberto e que exigem colaboração e troca entre pares se revelam promissoras em termos de evocação do pensamento metacognitivo, particularmente pela possibilidade de explicitação daquilo que os sujeitos estão compreendendo. Na pesquisa os autores investigaram o envolvimento metacognitivo de quatro grupos de estudantes do primeiro ano do Ensino Médio em Física (Cinemática) envolvendo ações em um parque de diversões. Os estudantes trabalharam em grupos para resolver coletivamente problemas de Física e foi analisado a partir desse trabalho coletivo, das interações, conversas e disposições comportamentais, a metacognição individual dos participantes. $\mathrm{O}$ foco estava em oportunizar que a partir do trabalho coletivo os alunos empregassem estratégias de revisões e reflexão para gerenciar sua própria compreensão, bem como processos de aprendizagem. A análise dos dados esteve atrelada à existência de três possibilidades de ativação metacognitiva durante as tarefas de aprendizagem em grupo: no estabelecimento de consensos decorrente da pesquisa colaborativa; no discurso argumentativo; nos momentos de discussão decorrentes das divergências do grupo. De acordo com os autores, em todo o processo houve evidências da evocação da metacognição em variados graus. Os novos problemas de Física abordados pelos alunos criaram situações em que discrepâncias entre seu conhecimento prévio e as experiências diretas permitindo que explicassem seu pensamento por meio de disposições de comportamento.

Saad et al. (2012), tratando mais diretamente da regulação da aprendizagem em contexto social, particularmente o da escola, mostram que tais processos requerem 
olhar para o que se está regulando e quem está sendo regulado. Em outras palavras, há uma diferença entre o que será regulado e em relação a quem se está regulando. No primeiro caso, pode-se estar regulando a atividade a ser realizada, como controlar para que as ações a serem desenvolvidas levem à utilização do pensamento metacognitivo, mas dentro de um processo de interação e de troca, ou, ainda, para que as situações sejam compartilhadas entre os estudantes. O segundo caso, o de quem se está regulando, é mais complexo e exige, como apontam Saab, van Joolingen e van Hout-Wolters (2012), uma análise em três momentos ou níveis: estar regulando a ação em nível individual (regulação intrapessoal - o estudante reflete sobre sua cognição), em nível pedagógico-didático (professor e estudante compartilham a regulação de forma que o aluno vá aos poucos se tornando autônomo) e em nível de grupo (regulação intrapessoal - troca entre os estudantes em uma espécie de aprendizagem colaborativa).

A metacognição social constituída a partir da perspectiva social da aprendizagem e seus avanços em relação aos estudos inicialmente apresentados por Flavell e Brown ainda se revelam incipientes na literatura, todavia, a sua possibilidade tem sido aventada desde a década de 1990, particularmente com os estudos da pesquisadora francesa Bernadette Noël. Embora não esteja voltado para a área de Ensino de Ciências seus estudos são importantes de serem mencionados, pois sua linha de pesquisa atribuía ao conceito de metacognição o entendimento de representar a "consciência necessária para a capacidade de verbalizar a ação e para fazer um julgamento durante o curso de uma atividade" (NOËL, 1991, p. 10, tradução nossa). Essa verbalização e a argumentação passam a ser pontos de análise dos pesquisadores como favorecedores da ativação do pensamento metacognitivo, realçando o entendimento de que a aprendizagem representa um fenômeno eminentemente social.

A argumentação e a metacognição não representam uma novidade no meio educacional. Pesquisadores como Osborne (2012) e Thomas (2012) têm indicado a pertinência dessa aproximação teórica, todavia, poucos são os estudos que mostram situações didáticas em que pelo uso da argumentação o pensamento metacognitivo é ativado. Um exemplo de estudo envolvendo situação didática foi o de Sanchéz-Castaño, Castaño-Mejía e Tamayo-Alzate (2015), desenvolvido com estudantes de 14 a 16 anos, na Colômbia, a partir de uma ação didática na abordagem CTS (Ciência-Tecnologia-Sociedade) que envolvia debates sobre: patente de medicamentos; dias em que não era possível usar o carro para transporte; vegetais transgênicos. A partir 
de uma metodologia envolvendo leitura e discussões em sala de aula, os autores de posse das gravações e transcrições das falas, categorizaram a argumentação metacognitiva em três situações vinculadas aos estudantes: no sentir-pensar-atuar; no conhecimento prévio dos temas; na postura ética. Por fim, os autores apontaram que essa reflexão metacognitiva foi possível uma vez que a proposta pedagógica permitiu que eles planejassem, exercitassem e avaliassem seus argumentos, aspectos tipicamente metacognitivos, segundo o texto.

Por fim, registramos que, nos estudos de intervenção pedagógica, independentemente da vertente individual ou social, de uso explícito ou implícito do pensamento metacognitivo, encontramos pesquisas - como a realizada por Sandi-Urena, Cooper e Stevens (2011) - que, ao cabo, não relatam aumento no uso do pensamento metacognitivo. Ou, ainda, há trabalhos que revelam um progresso nas habilidades metacognitivas demonstrado por alguns alunos após a intervenção, enquanto outros não observam esse avanço, como é o caso do estudo desenvolvido por Thomas e McRobbie (2001). Isso vem ao encontro da constatação de Veenman (2012), ao relatar a necessidade de haver mais estudos empíricos, anunciando que a pesquisa sobre metacognição na educação científica ainda é um trabalho em andamento.

\section{Considerações finais}

Por meio do que trouxemos neste artigo, procuramos elucidar que o campo da Educação, mais especificamente a área de Ensino de Ciências, tem se servido dos estudos em metacognição, retratado neste texto por discussões envolvendo situações que se passaram em sala de aula. Ao mesmo tempo em que os estudos apontam a existência de uma aproximação da metacognição com o ensino de Ciências pelo viés da pesquisa, o apresentado revelou carências e alternativas de novas perspectivas de investigação. Além do fato que a metacognição apresenta características significativas para o estabelecimento de ações que se aproximam do fazer científico, necessárias ao ensino e à aprendizagem em Ciências.

Dentre os aspectos revelados por esse relato de estudos - e tributo à metacognição - e que vem ao encontro do que expressam Rosa e Meneses Villagrá (2018), é o de que grande parte das pesquisas voltadas à intervenção pedagógico-didática focam na compreensão de que a metacognição está vinculada a uma reflexão de pensamento, sem, contudo, especificar características dessa reflexão. As que espe- 
cificam acabam por se ocupar com os processos vinculados à operacionalização dos componentes metacognitivos e seus elementos, traduzidos como habilidades para planejar, monitorar e avaliar a ação executada. Esse entendimento acaba por limitar a potencialidade do campo da metacognição, uma vez que é na integração dos diferentes componentes (conhecimentos, habilidades e experiências metacognitivas) que estão os benefícios para a aprendizagem, como mencionados por Flavell (1979).

Outra característica presente nas pesquisas analisadas é a limitação nas discussões sobre que aspectos da aprendizagem são favorecidos durante as ações didáticas orientadas explícita ou implicitamente pela metacognição. Os estudos, muitas vezes, limitam-se a apresentar que a instrução pedagógica favoreceu a ativação do pensamento metacognitivo - o que constitui um achado importante, todavia, poucos são os que estabelecem uma relação com a aprendizagem específica dos conteúdos. Esse achado vem ao encontro do identificado na revisão de estudo realizado por Avargil, Lavi e Dori (2018) no qual os autores relatam que as pesquisas sobre os processos de intervenção associadas à metacognição não são claras em identificar os elementos específicos de metacognição que foram contemplados, tanto nas construções teóricas específicas de regulação da cognição como no conhecimento/ conscientização da cognição.

Em termos da avaliação das contribuições das ações didáticas para a ativação do pensamento metacognitivo, assim como à aprendizagem em Ciências, precisamos considerar como destacado por Georghiades (2004) que a metacognição é processo interno e de difícil exteriorização pelos sujeitos. Isso, naturalmente, tem contribuído para as dificuldades encontradas para identificar e avaliar a presença dessa forma de pensamento nos estudos realizados na área de Ensino de Ciências. Situação que se intensifica quando analisamos uma situação coletiva com é a sala de aula. No entanto, como mencionado por Avargil, Lavi e Dori (2018), se alguém considerar a metacognição como um entendimento do conhecimento, então pode-se detectá-lo no aluno de forma indireta, quer por meio do uso efetivo desse entendimento, conforme testemunhado pelo comportamento do aprendiz, ou perguntando ao aluno que forneça uma descrição explícita.

Contudo, é importante destacar ao final deste texto que mesmo diante das limitações mencionadas, os estudos revelam resultados promissores, embora autores como Veenman (2012) mencionem a necessidade de ampliar a gama de dados empíricos 
sobre as contribuições das instruções pedagógicas direcionadas especificamente à ativação dessa forma de pensamento.

Como perspectivas emergentes incluímos, ao final deste tributo comemorativo aos 50 anos de pesquisas em metacognição - (1971-2021) -, a necessidade de ampliar os estudos, particularmente, aqueles associados à componente social, com realce ao papel da argumentação como coadjuvante na ativação do pensamento metacognitivo. Tais escolhas de atuação, caminhos a serem trilhados, desejos em realização, representam uma nova enseada nas investigações sobre metacognição para a área de Ensino de Ciências e, por conseguinte, o aprendizado de Ciências em sala de aula.

\section{Metacognition and its 50 years: scenarios and perspectives for Science Teaching}

\section{Abstract}

Studies involving metacognition complete 50 years and reveal a diversity of areas that have been using this concept that was coined by Flavell in the 1970s. In addition, and closer to the educational context, metacognition has been identified as a proposal that contributes to learning, which has provided an opportunity to look more closely at the metacognitive components (and their respective elements). Considering the possibility of paying tribute to John Hurley Flavell who published his first article in 1971 presenting the term metamemory that gave rise to the name metacognition, we elaborated this article. In it we disclose a set of studies that address the use of metacognition in science classrooms, presenting the characteristics and impacts of these practices, as well as bringing new possibilities for studies and interventions. For this: we introduced the term historically and some details about the first investigations carried out; we describe several reflections suggested by those who sought to advance the understanding and application of metacognition in numerous areas; we list results of research carried out in classrooms; we highlight the possibilities of choices and practicable paths for a Science Teaching based on metacognitive experiences.

Keywords: John Hurley Flavell; Metacognition; Science education.

\section{Nota}

1 O termo "expert" é apontado nas pesquisas em metacognição como o sujeito que retém as características corretas da situação problema, que seleciona estratégias adequadas e as utiliza de maneira certa, ou, mesmo, considera-se aquele que aprende de forma mais eficiente, apresentando maior facilidade na apropriação dos conhecimentos. Em contrapartida, tem-se o "novato" como aquele que ainda não atingiu o nível de compreensão desejado. 


\section{Referências}

ANDERSON, David; NASHON, Samson. Predators of knowledge construction: Interpreting students' metacognition in an amusement park physics program. Science Education, v. 91, n. 2, p. 298-320, 2007. https://doi.org/10.1002/sce.20176

AVARGIL, Shirly; LAVI, Rea; DORI, Yehudit Judy. Students' metacognition and metacognitive strategies in science education. In: DORI, Yehudit Judy; MEVARECH, Zemira R; BAKER, Dale R. (Eds.). Cognition, Metacognition, and Culture in STEM Education. The Netherlands: Springer-Verlag, 2018. p. 33-64. https://doi.org/10.1007/978-3-319-66659-4

BOGDANOVIC, Ivana; OBADOVIC, Dusanka Z.; CVJETICANIN, Stanko; SEGEDINAC, Mirjana; BUDIC, Spomenka. Students' metacognitive awareness and physics learning efficiency and correlation between them. European Journal of Physics Education, v. 6, n. 2, p. 18-30, 2017.

BOSZKO, Camila; ROSA, Cleci T. Werner da. Diários de aprendizagem como ferramenta metacognitiva: análise dos registros produzidos por professores de Física em formação inicial. Alexandria: Revista de Educação em Ciência e Tecnologia, 2020. (prelo).

BROWN, Ann L. Knowing when, where, and how to remember: a problem of metacognition. In: GLASER, Robert. (Ed.). Advances in Instructional Psychology. New Jersey: LEA, 1978. p. 77-165.

CHI, Michelene T. H.; BASSOK, Miriam; LEWIS, Matthew W.; REIMANN, Peter; GLASER, Robert. Self-explanations: How students study and use examples in learning to solve problems. Cognitive Science, v. 13, n. 2, p. 145-182, 1989.

CHI, Michelene T. H.; GLASER, Robert; REES, Ernest. Expertise in problem solving. In: STERNBERG, Robert J. (Ed.). Advances in the psychology of human intelligence. New Jersey: Erlbaum, 1982. p. 7-75.

CORREAA, Nancy N. G.; PASSOS, Marinez M.; ARRUDA, Sergio M. Metacognição e as relações com o saber. Ciência \& Educação, v. 24, n. 2, p. 517-534, 2018a. https://doi.org/10.1590/1516731320180020016

CORRÊA, Nancy N. G.; PASSOS, Marinez M.; ARRUDA, Sergio M. Perfil metacognitivo (Parte II): aplicação de instrumento de análise. Investigações em Ensino de Ciências, v. 23, n. 1, p. 176-191, 2018b. http://dx.doi.org/10.22600/1518-8795.ienci2018v23n1p230

CORREAA, Nancy N. G.; PASSOS, Marinez M.; CORREAA, Hugo E. R. ARRUDA, Sergio M. Estudo exploratório sobre o uso da palavra "metacognição" em artigos publicados em periódicos brasileiros do ensino de ciências e matemática de 2007 a 2017. Caderno Brasileiro de Ensino de Física, v. 37, n. 1, p. 6-26, 2020. https://doi.org/10.5007/2175-7941.2020v37n1p6

EILAM, Billie; REITER, Shoshi. Long-term self-regulation of biology learning using standard junior high school science curriculum. Science Education, v. 98, n. 4, p. 705-737, 2014.

FLAVELL, John H. First discussant's comments: what is memory development the development of? Human Development, v. 14, n. 4, p. 272-278, 1971. https://doi.org/10.1159/000271221

FLAVELL, John H. Metacognition and cognitive monitoring. American Psychologist, v. 34, n. 10, p. 906-911, 1979. http://dx.doi.org/10.1037/0003-066X.34.10.906

FLAVELL, John H. Metacognitive aspects of problem solving. In: RESNICK, Lauren B. (Ed.). The nature of intelligence. New Jersey: LEA, 1976. p. 231-236. 
GEORGHIADES, Petros. From the general to the situated: Three decades of metacognition. International Journal of Science Education, v. 26, n. 3, p. 365-383, 2004. https://doi. org/10.1080/0950069032000119401

GROTZER, Tina; MITTLEFEHLDT, Sarah. The role of metacognition in students' understanding and transfer of explanatory structures in science. In: ZOHAR, Anat; DORI, Yehudit J. (Eds.). Metacognition in science education: trends in current research. Dordrecht: Springer, 2012. p. 79-99.

GUNSTONE, Richard F. The importance of specific science content in the enhancement of metacognition. In: FENSHAM, Peter J.; GUNSTONE, Richard F.; WHITE, Richard T. (Eds.). The content of science: a constructivist approach to teaching and learning. Washington: Falmer, 1994. p. 131-146.

HACKER, Douglas J. Definitions and empirical foundations. In: HACKER, Douglas J.; DUNLOSKY, John; GRAESSER, Arthur C. Metacognition in educational theory and practice. New Jersey: LEA, 1998. p. 15-38.

HATTIE, John A. C. Visible learning: a synthesis of over 800 meta-analyses relating to achievement. New York: Routledge, 2009.

HUANG, Kun; GE, Xun; ESERYEL, Deniz. Metaconceptually-enhanced simulation-based inquiry: effects on eighth grade students' conceptual change and science epistemic beliefs. Educational Technology Research and Development, v. 65, n. 1, p. 75-100, 2017. https://doi.org/10.1007/ s11423-016-9462-5

HUFF, Jessica D.; NIETFELD, John L. Using strategy instruction and confidence judgments to improve metacognitive monitoring. Metacognition and Learning, v. 4, n. 2, p. 161-176, 2009. https://doi.org/10.1007/s11409-009-9042-8

KALMAN, Calvin S.; SOBHANZADEH, Mandana; THOMPSON, Robert; IBRAHIM, Ahmed; WANG, Xihui. Combination of interventions can change students' epistemological beliefs. Physical Review Special Topics-Physics Education Research, v. 11, n. 2, p. 020136, 2015. https:// doi.org/10.1103/PhysRevSTPER.11.020136

KISTNER, Saskia; RAKOCZY, Katrin; OTTO, Barbara; DIGNATH-VAN EWIJK, Charlotte; BUTTNER, Gerhard; KLIEME, Eckhard. Promotion of self-regulated learning in classrooms: investigating frequency, quality, and consequences for student performance. Metacognition and Learning, v. 5, n. 2, p. 157-171, 2010. https://doi.org/10.1007/s11409-010-9055-3

$\mathrm{KOCH}$, Adina. Training in metacognition and comprehension of physics texts. Science Education, v. 85, n. 6, p. 758-768, 2001. https://doi.org/10.1002/sce.1037

LARKIN, Jill H. The role of problem representation in physics. In: GENTNER, Dedre; STEVENS, Albert L. (Eds.). Mental Models. New Jersey: LEA, 1983. p. 75-98.

MARTINEZ, Michael E. What is metacognition? Phi delta kappan, v. 87, n. 9, p. 696-699, 2006. https://doi.org/10.1177/003172170608700916

MEDEIROS, Edilene F.; SILVA, Marcia G. L.; LOCATELLI, Solange W. A argumentação e o potencial metacognitivo de uma atividade experimental baseada na POA (Previsão-Observação-Argumentação). Amazônia: Revista de Educação em Ciências e Matemáticas, v. 14, n. 29, p. 27-42, 2018. 
MONEREO, Carlos. La enseñanza estratégica: enseñar para la autonomía. In: MONEREO, Carlos (Org.). Ser estratégico y autónomo aprendiendo. Barcelona: Graó, 2001. p. 11-27.

NELSON, Thomas O.; NARENS, Louis. Why investigate metacognition. In: JANET, Metcalfe; SHIMAMURA, Arthur P. (Org.). Metacognition: knowing about knowing. Cambrigde: ABB, 1994. p. 1-25.

NETO, Antonio; VALENTE, Maria O. Problem Solving in Physics: Towards a Metacognitive ly Developed Approach, 1997. Disponível em: https:/files.eric.ed.gov/fulltext/ED405217.pdf. Acesso em: 28 jan. 2017.

NIELSEN, W. S., NASHON, Samson; ANDERSON, David. Metacognitive engagement during fieldtrip experiences: A case study of students in an amusement park physics program. Journal of Research in Science Teaching, v. 46, n. 3, p. 265-288, 2009. https://doi.org/10.1002/tea.20266

NOËL, Bernadette. La métacognition. Bruxelles: De Boeck Université, 1991.

OSBORNE, Jonathan. The rol of Argument: Learning how to learn in School Science. In: FRASER, Barry J.; TOBIN, Kenneth; MCROBBIE, Campell J. (Eds.). Second International Handbook of Science Education. New York: Springer, 2012. p. 933-949.

PASSOS, Marinez M.; CORRÊA, Nancy N. G.; ARRUDA, Sérgio M. Perfil metacognitivo (parte I): uma proposta de instrumento de análise. Investigações em Ensino de Ciências, v. 22, n. 3, p. 176-191, 2017.

PETERS-BURTON, Erin E. Developing content knowledge in students through explicit teaching of the nature of science: Influences of goal setting and self-monitoring. Science \& Education, v. 21 , n. 6 , p. 881-898, 2012. https://doi.org/10.1007/s11191-009-9219-1

PHILLIPS, Anna M.; WATKINS, Jessica; HAMMER, David. Beyond “asking questions": Problematizing as a disciplinary activity. Journal of Research in Science Teaching, v. 55, n. 7, p. 982-998, 2018. https://doi.org/10.1002/tea.21477

ROSA, Cleci T. Werner da. A metacognição e as atividades experimentais no ensino de Física. 2011. 324f. Tese (Doutorado em Educação Científica e Tecnológica) - Universidade Federal de Santa Catarina, Florianópolis, 2011.

ROSA, Cleci T. Werner da; MENESES VILLAGRÁ, Jesús Á. Metacognição e ensino de Física: revisão de pesquisas associadas a intervenções didáticas. Revista Brasileira de Pesquisa em Educação em Ciências, v. 18, n. 2, p. 581-608, 2018. https://doi.org/10.28976/1984-2686rbpec2018182581

RYAN, Qing X.; FRODERMANN, Evan; HELLER, Kenneth; HSU, Leonardo; MASON, Andrew. Computer problem-solving coaches for introductory physics: Design and usability studies. Physical Review Physics Education Research, v. 12, n. 1, p. 0101051-17, 2016. https://doi. org/10.1103/PhysRevPhysEducRes.12.010105

SAAB, Nadira; JOOLINGEN, Wouter van; HOUT-WOLTERS, Bernadette van. Support of the collaborative inquiry learning process: Influence of support on task and team regulation. Metacognition and Learning, v. 7, n. 1, p. 7-23, 2012. https://doi.org/10.1007/s11409-011-9068-6

SÁNCHEZ-CASTAÑO, Jairo A.; CASTAÑO-MEJÍA, Olga Y.; TAMAYO-ALZATE, Óscar E. La argumentación metacognitiva en el aula de ciencias. Revista Latinoamericana de Ciencias Sociales, Niñez y Juventud, v. 13, n. 2, p. 1153-1168, 2015. 
SANDI-URENA, Santiago; COOPER, Melanie M.; STEVENS, Ron H. Effect of cooperative problem-based lab instruction on metacognition and problem-solving skills. Journal of Chemical Education, v. 89, n. 6, p. 700-706, 2012. https://doi.org/10.1021/ed1011844

SANDI-URENA, Santiago; COOPER, Melanie M.; STEVENS, Ron H. Enhancement of metacognition use and awareness by means of a collaborative intervention. International Journal of Science Education, v. 33, n. 3, p. 323-340, 2011. https://doi.org/10.1080/09500690903452922

SARIBAS, Deniz; MUGALOGLU, Ebru Z.; BAYRAM, Hale. Creating Metacognitive Awareness in the Lab: Outcomes for Preservice Science Teachers. EURASIA: Journal of Mathematics, Science and Technology Education, v. 9, n. 1, p. 83-88, 2013. https://doi.org/10.12973/ eurasia.2013.918a

SCHRAW, Gregory; CRIPPEN, Kent J.; HARTLEY, Kendall. Promoting self-regulation in science education: Metacognition as part of a broader perspective on learning. Research in Science Education, v. 36, n. 1-2, p. 111-139, 2006. https://doi.org/10.1007/s11165-005-3917-8

TAASOOBSHIRAZI, Gita; FARLEY, John. A Multivariate Model of Physics Problem Solving. Learning and Individual Differences, v. 24, p. 53-62, 2013. https://doi.org/10.1016/j.lindif.2012.05.001

TAMAYO-ALZATE, Oscar E. La metacognición en los modelos para la enseñanza y el aprendizaje de las ciencias. In: BOOM, Alberto M. (Ed.). Los bordes de la pedagogía: del modelo a la ruptura. Bogotá: Universidad Pedagógica Nacional, 2006. p. 275-306.

THOMAS, Gregory P. Changing the metacognitive orientation of a classroom environment to stimulate metacognitive reflection regarding the nature of physics learning. International Journal of Science Education, v. 35, n. 7, p. 1183-1207, 2013. https://dx.doi.org/10.1080/095 00693.2013.778438

THOMAS, Gregory P. Metacognition in Science Education: Past, Present and Future Considerations. In: FRASER, Barry J.; TOBIN, Kenneth; MCROBBIE, Campell J. (Eds.). Second International Handbook of Science Education. New York: Springer, 2012. p. 131-144.

THOMAS, Gregory P.; MCROBBIE, Campell J. Using a metaphor for learning to improve students' metacognition in the chemistry classroom. Journal of Research in Science Teaching, v. 38, $n$. 2, p. 222-259, 2001. https://doi.org/10.1002/1098-2736(200102)38:2<222::AID-TEA1004>3.0.CO;2-S

THOMAS, Gregory P.; MEE, Doris A. K. Changing the learning environment to enhance students' metacognition in Hong Kong primary school classrooms. Learning Environments Research, v. 8, n. 3, p. 221-243, 2005. https://doi.org/10.1007/s10984-005-1565-6

VEENMAN, Marcel V. J. Metacognition in science education: definitions, constituents, and their intricate relation with cognition. In: ZOHAR, Anat; DORI, Yehudit J. (Eds.). Metacognition in science education: trends in current research. Dordrecht: Springer, 2012. p. 21-36.

VERPOORTEN, Dominique; CASTAIGNE, Jean-Loup; WESTERA, Wim; SPECHT, Marcus. A quest for meta-learning gains in a physics serious game. Education and Information Technologies, v. 19, n. 2, p. 361-374, 2014. https://doi.org/10.1007/s10639-012-9219-7

WANG, Jing-Ru; CHEN, Shin-Feng. Exploring mediating effect of metacognitive awareness on comprehension of science texts through structural equation modeling analysis. Journal of Research in Science Teaching, v. 51, n. 2, p. 175-191, 2014. 
WEINERT, Franz E. Metacognition and motivation as determinants of effective learning and understanding. In: WEINERT, Franz E.; KLUWE, Rainer H. (Eds.). Metacognition, motivation, and understanding. New Jersey: LEA, 1987. p. 1-16.

YURUK, Nejla; BEETH, Michael E; ANDERSEN, Christopher. Analyzing the effect of metaconceptual teaching practices on students' understanding of force and motion concepts. Research in Science Education, v. 39, n. 4, p. 449-475, 2009. https://doi.org/10.1007/s11165-008-9089-6

ZOHAR, Anat; BARZILAI, Sarit. A review of research on metacognition in science education: current and future directions. Studies in Science Education, v. 49, n. 2, p. 121-169, 2013. https://doi.org/10.1080/03057267.2013.847261 